\title{
Morphologische Verbfehler in elizitierten Narrativen bei russisch-deutsch bilingualen Kindern im Grundschulalter
}

\author{
Morphological verb errors in elicited narratives of Russian-German \\ primary school bilinguals
}

\author{
Olga Vorobyeva \\ Humboldt-Universität zu Berlin, Deutschland
}

This paper focuses on morphological verb errors in elicited narratives of RussianGerman primary school bilinguals. The data was collected from 37 children who were separated into four groups according to the age and language acquisition type (simultaneous and successive). The Multilingual Assessment Instrument for Narratives (MAIN) (Gagarina et al. 2012) was used for data collection. The narratives produced in mode telling after listening to a model story were analysed and morphological verb errors in Russian and German were classified. Therefore, the error classification of Gagarina (2008) for Russian monolingual children was expanded and for the classification of German errors an own classification was suggested. Errors in Russian typically produced by monolinguals and unique bilingual errors as well were documented. The results show that the language of the environment (German) increases with age. Older children make fewer errors than younger ones. Nevertheless, a strong heterogeneity between children within each group can be observed.

\section{$1 \quad$ Einleitung}

Die sprachliche Entwicklung eines mehrsprachigen Kindes ist von vielen Faktoren geprägt und stellt sowohl für die Bildungseinrichtungen als auch für die Eltern eine große Herausforderung dar. Zur Erfassung der Sprachkompetenz eines mehrsprachig aufwachsenden Kindes gehört neben der Bewertung seiner Kenntnisse im Deutschen auch der Leistungsstand in seiner Herkunftssprache. Fehlerproduktion ist ein unabdingbarer Prozess im Spracherwerb. In der Kindersprache kommen oft Fehler vor, die als sprachliche Probehandlungen zum Überprüfen ihrer Hypothesen über bestimmte sprachliche Erscheinungen dienen. 
Während des Spracherwerbsprozesses machen Kinder Fehler im Wortgebrauch und bilden innovative Wortformen.

Ziel dieser Studie ist es, in elizitierten Narrativen deutsch-russischer Kinder, die in beiden Sprachen erzählt werden, morphologische Verbfehler zu finden und zu klassifizieren. Speziell soll das Vorkommen sogenannter bilingual unique errors geprüft werden, die bei monolingualen Kindern nicht auftreten. Solche Einflussfaktoren auf die Sprachentwicklung, wie Alter und Kontaktzeit mit beiden Sprachen, sollen analysiert werden.

\section{Spracherwerb bei bilingualen Kindern}

Bei einem regelmäßigen Input in der Umgebungssprache beginnt der Erstspracherwerb bei jedem normal entwickelten Kind ohne Unterweisung (vgl. Tracy 2002:3). In der vorliegenden Studie wird vom Bilingualismus ${ }^{1}$ nach der Definition von Tracy/Gawlitzek-Maiwald (2000:497) ausgegangen:

Ein bilinguales Individuum beherrscht zwei sprachliche Kenntnissysteme in einem Ausmaß, das es ihm gestattet, mit monolingualen Sprechern der einen oder der anderen Sprache in einem „monolingualen Modus“, d.h. in der Sprache des Gesprächspartners zu kommunizieren. Bei Bedarf, d.h. im Umgang mit mehrsprachigen Kommunikationspartnern, kann sich ein bilinguales Individuum der Ressourcen des „bilingualen“ Modus bedienen, d.h. ein beide sprachen umfassendes Repertoire ausschöpfen, wobei es zu intensiven Formen des Mischens oder Code-switching kommen kann.

Die relevante Altersgrenze ist in der bisherigen Forschungslage umstritten. De Houwer (1990:2ff) spricht nur dann vom doppelten Erstspracherwerb (oder simultanen Bilingualismus, auch 2L1-Erwerb), wenn das Einstiegsalter innerhalb der ersten Wochen nach der Geburt liegt und das Kind ein tägliches Angebot in beiden Sprachen bekommt. Tracy/Gawlitzek-Maiwald (2000:503) schlagen vor, vom simultanen Bilingualismus zu sprechen, wenn das Kind in den ersten beiden Lebensjahren in intensiven Kontakt mit beiden Sprachen tritt und einen regelmäßigen Input bekommt. In einigen Studien wird davon ausgegangen, dass der simultan bilinguale Spracherwerb ebenso wie der monolinguale abläuft, wenn der Erwerbsbeginn vor dem dritten Lebensjahr liegt (McLaughlin 1984:101, Anstatt/Dieser 2007:140, Montrul 2008:94, Paradis et al. 2011:59). Einige

1 Die Begriffe Bilingualismus und Zweisprachigkeit werden in der vorliegenden Arbeit synonym verwendet. 
Wissenschaftler ziehen die Grenze beim Einstiegsalter in den ersten vier Jahren (vgl. Genesee 1989).

Vom sukzessiven Bilingualismus (auch Zweitspracherwerb oder L2Erwerb) wird gesprochen, wenn mit dem Erwerb einer zweiten Sprache begonnen wird, nachdem die linguistischen Grundlagen der Erstsprache (L1) etabliert wurden (vgl. Montrul 2008:97, Paradis et al. 2011:109). Auch wenn hier die Altersgrenze variiert, unterscheiden die meisten Autoren zwischen dem frühen bzw. kindlichen Zweitspracherwerb (abgekürzt cL2 mit $c$ für child), der mit einem regelmäßigen Input in der Zweitsprache bis zum siebten/achten Lebensjahr beginnt (vgl. Meisel 2007:104) und dem späteren bzw. erwachsenen Zweitspracherwerb (abgekürzt aL2 mit $a$ für adult). In einigen Studien spricht man vom späten Zweispracherwerb erst ab dem 10. Lebensjahr (Anstatt/Dieser 2007:141). Bei einem sukzessiven Spracherwerb werden die Kinder zunächst einsprachig in ihrer Familie erzogen und erwerben die Zweitsprache, wenn sie in eine weitere soziale Umwelt kommen, in der diese Sprache gesprochen wird. (Kreppel:29).

Ruberg (2013:182) unterscheidet vier Typen des zwei- und mehrsprachigen Erwerbs in Abhängigkeit vom Alter bei Erwerbsbeginn. Beim Erwerbsbeginn im 1. - 2. Lebensjahr $\left(\mathrm{AoO}^{2} 0 ; 0\right.$ bis 1;11 Jahre) spricht er vom simultanen bilingualen Erwerb, beim Erwerbsbeginn im 3. - 4. Lebensjahr (AoO 2;0 bis 3;11 Jahre) spricht er vom sukzessiven bilingualen Erwerb. Beim Erwerbsbeginn im 5. - 10. Lebensjahr (AoO 4;0 bis 9;11 Jahre) wird vom kindlichen Zweitspracherwerb gesprochen und beim Erwerbsbeginn ab dem 11. Lebensjahr (AoO ab 10;0) vom erwachsenen Zweitspracherwerb.

In der vorliegenden Arbeit wird die Altersgrenze zwischen dem simultan bilingualen und sukzessiv bilingualen Spracherwerb auf das Alter von 2;0 festgelegt: beim AoO der L2 von 0;0 bis $1 ; 11$ werden Kinder als simultan bilingual bezeichnet, beim AoO der L2 ab 2;0 wird von sukzessiv bilingualen Kindern gesprochen.

\section{Erwerb der grammatischen Kategorien der Verben}

\subsection{Erwerb der grammatischen Kategorien der Verben im Russischen}

Im Russischen beginnt der Erwerb des Verblexikons und der Verbmorphologie ab einem Alter von 1;9 (Gagarina 2011:147). Dabei zeigen die Untersuchungen von bilingualen Kindern im Vergleich zu monolingualen, dass die Herausbildung des produktiven Gebrauchs der Verbflexion bei jedem Kind nach dem Erwerb des

2 AoO (Age of Onset $)=$ Alter beim Erwerbsbeginn. 
ersten Verbs in unterschiedlich langen Zeitabschnitten erfolgt, die bei monolingualen Kindern kürzer sind (vgl. ebd.:148).

Im Allgemeinen verläuft der Erwerb der Verbflexion im Russischen bei bilingual aufwachsenden Kindern sehr ähnlich wie bei monolingualen (vgl. ebd.), nimmt aber in der Regel etwas mehr Zeit in Anspruch (vgl. ebd:156, Anstatt 2006:21). ${ }^{3}$

\subsubsection{Aspekt}

Dem Aspekterwerb im Russischen bei bilingualen Kindern wird in der Forschung viel Aufmerksamkeit gewidmet (u.a. Anstatt 2006 u. 2008, Gagarina 2008 u. 2011). In diesen Studien stellt sich heraus, dass bilinguale Kinder mit L1 Russisch Probleme beim Gebrauch der Aspektpaare aufweisen, wobei sie öfters imperfektive Verben anstelle von perfektiven gebrauchen. Dabei handelt es sich um unique bilingual errors des Aspekts, da bei monolingualen Kindern nur der Gebrauch perfektiver Verben anstelle von imperfektiven dokumentiert wurde (Gagarina 2011:142).

Der Erwerb des Verbalaspekts - einer grammatischen Kategorie, die das Deutsche nicht kennt - verläuft bei bilingualen Kindern sehr heterogen und auch, wenn sie im Durchschnitt mehr Fehler als monolinguale Kinder machen, lassen sich bei einigen Kindern viele Aspektfehler, bei den anderen aber gar keine feststellen (Anstatt 2008a:19ff). Jedoch besitzt die Kategorie des Verbalaspekts eine Indikatorfunktion: Eine sehr hohe Anzahl von Modifikationen des Aspekts pro 100 Verbtokens geht immer einher mit einer sehr hohen Anzahl von Modifikationen insgesamt und ist in der Regel ein Hinweis auf insgesamt schwach ausgeprägte aktive Sprachfähigkeit (Anstatt 2009:129).

\subsubsection{Genus}

Der Genuserwerb von ein- und mehrsprachigen Kindern wird ausführlich von Dieser (2009) untersucht. Im Russischen gibt es drei Genera (Maskulinum, Femininum und Neutrum) und neben den Substantiven, Adjektiven und Pronomina weisen im Russischen, im Gegensatz zum Deutschen, auch Partizipien und Verben Genuskongruenz auf (vgl. Dieser 2009:39). Monolingual aufwachsende Kinder beherrschen das Genussystem des Russischen kurz vor der Einschulung oder sogar erst in den ersten Grundschuljahren komplett (ebd.:49). Bei bilingual aufwachsenden Kindern nimmt der Genuserwerbsprozess unterschiedlich viel Zeit in Anspruch und ist sehr heterogen. Laut Ergebnissen

\footnotetext{
${ }^{3}$ mehr zum L1-Erwerb der grammatischen Kategorien s. Gagarina (2008:60ff)
} 
von Dieser (ebd.:166) erreichen einige bilinguale Kinder das Niveau von monolingualen Kindern ebenfalls um das 7. Lebensjahr, die meisten beherrschen das Genussystem des Russischen erst zwischen dem 10. und dem 14. Lebensjahr, andere sogar noch später.

Bei der Frage des gegenseitigen Einflusses der einen Sprache auf die andere stellt Anstatt (ebd.) fest, dass bei simultan bilingualen Kindern der Einfluss der Umgebungssprache (im Untersuchungsfall des Deutschen) stärker und qualitativ abwechslungsreicher als bei sukzessiv bilingualen Kindern ausgeprägt ist.

Simultan bilinguale Kinder machen im Durchschnitt mehr Fehler im Russischen als sukzessiv bilinguale Kinder, allerdings verläuft der Spracherwerb bei jedem Kind individuell (vgl. ebd.).

Protassova (2011:290f) kommt in ihrer Studie, in der sie Narrative von russisch-finnisch bilingualen und monolingualen Kindern analysiert, zum Ergebnis, dass im Durchschnitt gleichaltrige bilinguale und monolinguale Kinder gleiche Ergebnisse zeigen. Einige von der Autorin untersuchte bilinguale Kinder sprechen zum Teil weniger als gleichaltrige monolinguale und machen dadurch auch weniger Fehler als sie.

\subsection{Erwerb der grammatischen Kategorien der Verben im Deutschen}

Bei monolingualen Kindern wird die Subjekt-Verb-Kongruenz mit etwa drei Jahren etabliert (Kauschke 2012:83). Es nimmt aber trotzdem eine längere Zeit in Anspruch, bis morphologisch markierte Formen fehlerfrei verwendet werden (ebd.). Der Erwerb von Verbflexionen ist eng mit der syntaktischen Entwicklung verknüpft und beginnt mit dem Auftreten von Mehrwortäußerungen mit Verben und schließt mit dem Auftreten der Flexion -st für die zweite Person Singular im Alter von 2;4 bis 3;0 ab (Kauschke 2012:80).

Im Tempuserwerb lassen sich bei monolingualen Kindern auch nach dem Alter von 3;0 noch lange Übergeneralisierungen, bei denen das $-t$-Suffix des Partizips des schwachen Verbs auf die starken übertragen wird, beobachten (Kauschke 2012:82).

Kostyuk (2005) beschreibt den Erwerb des Deutschen als Zweitsprache am Beispiel von drei russischprachigen Kindern im Alter zwischen 2;9 und 5;4 Jahren. Im Bereich der Verbmorphologie beschäftigt sie sich unter anderem mit der Erwerbsreihenfolge von verbalen Flexionen bei Kindern und untersucht, welche Tempusformen von Kindern bevorzugt werden (Kostyuk 2005:251). Bei den von ihr untersuchten Kindern lassen sich jedoch keine klaren Abgrenzungen für die Erwerbsreihenfolge verbaler Strukturen feststellen (ebd.:255). Für alle drei Kinder ist es typisch, dass ,sie zuerst die Verbflexion im Singular und später im Plural beherrschen" (ebd.:272) und bei allen drei Kindern beobachtet die Autorin, dass „sie den Infinitiv parallel mit flexionsmarkierten Formen gebrauchen“ 
(ebd.:446). Die erste von den drei Kindern erworbene Tempusform ist das Perfekt (ebd.:447). Aber während bei zwei Kindern häufig übergeneralisierte Partizipformen sowohl auf $-n$ (z.B. * gespielen) als auch auf - $t$ (z.B. *gefahrt) dokumentiert werden konnten, kamen bei einem Kind nur fünf Beispiele für Übergeneralisierungen bei der Bildung des Partizip Perfekts vor (ebd.: 273).

\section{Morphologische Fehler im Verbgebrauch}

\subsection{Morphologische Verbfehler im Russischen}

Sprachfehler monolingualer Kinder im Russischen wurden von Cejtlin (2013) detailliert untersucht (s.a. Cejtlin 2009 u. 2011). Die Autorin klassifiziert dabei form- und wortbildende Innovationen von Substantiven, Verben und Adjektiven in der Kindersprache. Im Verbgebrauch unterscheidet sie wortbildende Innovationen, die Wortneuschöpfungen und Formänderungen der normativen Sprache bilden, sowie morphologische Fehler, die mit der nicht normativen Formbildung und Wortgebrauch verbunden sind (Cejtlin 2013:25f).

Keine der kindlichen Wortneuschöpfungen widersprechen dem Wortbildungs-system der Sprache und werden sogar durch dieses bestimmt, indem sie die unerschöpflichen Möglichkeiten der Wortbildung zeigen. Das heißt, dass es sich bei jeder kindlichen Innovation um eine neue Morphemkombination handelt, wobei die Morpheme an sich, sowie ihre Verbindungsmittel nicht neu erfunden werden, sondern bereits existierende Elemente eines Sprachsystems sind, die neu miteinander kombiniert werden. Dabei entsprechen jedoch die Innovationen in der Kindersprache nicht der Norm, da sie bestimmte Sprachgesetze übertreten (Cejtlin 2013:31f) und somit als Fehler gelten.

Gagarina (2008:146) erstellt eine Klassifikation der morphologischen formbildenden Verbfehler, welche monolinguale Kinder machen, und unterteilt diese in zwei Gruppen (mit weiterer Unterteilung in Untergruppen) in Abhängigkeit des betroffenen Teils des morphologischen Systems: Flexionstyp (oder Marker der Verbkategorie) wie z.B. Person, Numerus und Tempus usw., oder der Stamm, der für die Formbildung vom Kind ausgesucht wird. Die Klassifikation ist in Abbildung 1 dargestellt. 


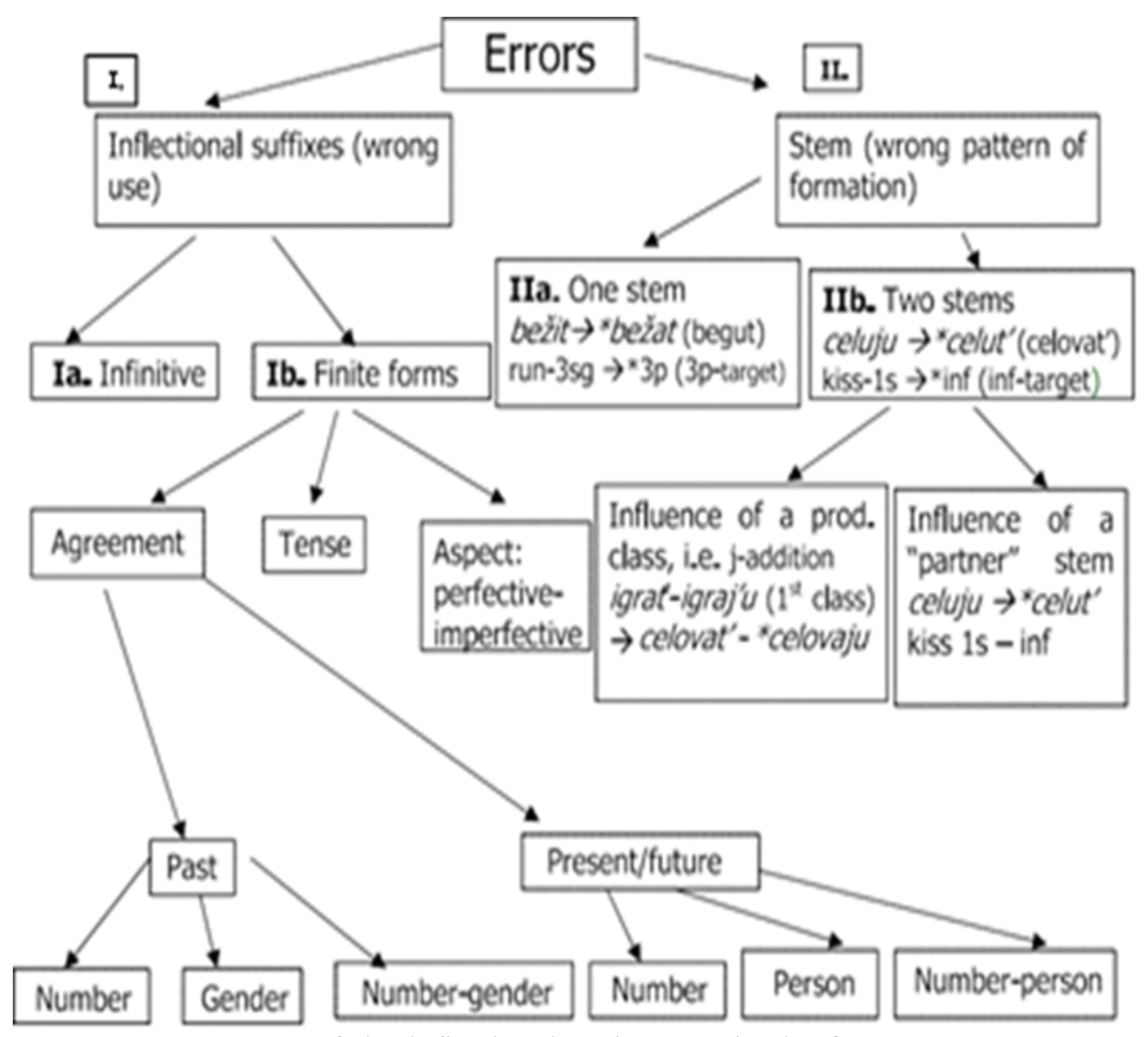

Abbildung 1: Types of the inflectional verb errors in the first language acquisition of Russian (Gagarina 2008:235)

Da bilinguale Kinder zusätzlich die sogenannten unique bilingual errors machen (vgl. Gagarina 2011:142), welche bei monolingualen Kindern nicht dokumentiert sind, ist es notwendig, die vorliegende Klassifikation zu erweitern, um sie auf bilinguale Kinder anwenden zu können. So wurde in der Fehlergruppe IIb. Zwei Stämme eine weitere Untergruppe hinzugefügt: Stammerweiterung durch Reflexionssuffix (Abb. 2), da dieses Suffix nach Panova (2010:21) zu den formbildenden gezählt wird und dieser Fehler bei zwei im Rahmen der vorliegenden Studie untersuchten Kindern vorkommt. Außerdem wurden in der Klassifikation von Gagarina (2008:235) Beispiele für jeden Fehlertyp hinzugefügt (s. Abb. 2). 


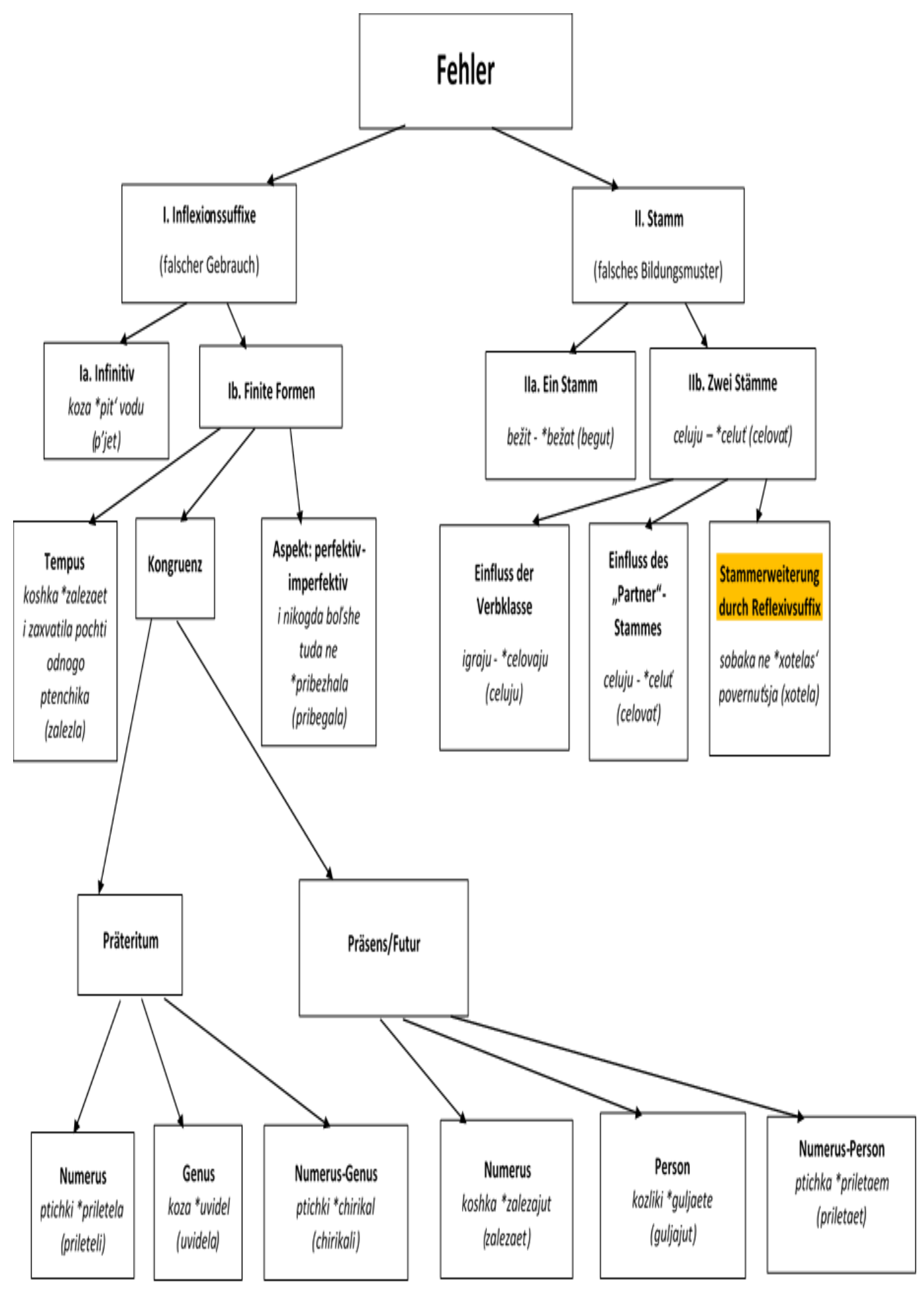

Abbildung 2: Klassifikation der morphologischen Verbfehler im Russischen

\subsection{Morphologische Verbfehler im Deutschen}

Unter den häufigsten morphologischen Verbfehlern nennt (Böttger 2008:131) den falschen Gebrauch des Tempus und Übergeneralisierungen im Gebrauch 
schwacher Verben, d.h. das Übertragen des Konjugationsmusters der schwachen Verben auf starke. Im Präteritum bekommen dabei die starken Verben die Endung -te $(* i c h$ fliegte, *ich denkte) und im Perfekt die Endung -t (*weggefahrt, * gekommt, *gebittet), die nach der Regel auf -en enden (ebd.:115). Solche Fehler wurden sowohl bei bilingualen Kindern dokumentiert (Kostyuk 2005:273), kommen aber auch sehr oft bei monolingualen Kindern vor (vgl. Ramge 1980:10, Kauschke 2012:82).

Auf Grundlage der morphologischen Kategorien der Verben im Deutschen nach Helbig/Buscha (2001) wird hier folgende Klassifikation der morphologischen Verbfehler im Deutschen vorgeschlagen (Abb. 3).

Alle Fehlertypen können in zwei Gruppen (mit Untergruppen) aufgeteilt werden. Die Gruppe I (Inflexionssuffixe) bilden Fehler, die einen falschen Gebrauch bereits existierender Verbformen darstellen. Diese Fehlergruppe wird in zwei Untergruppen unterteilt: Ia - Gebrauch von Infinitivformen anstelle von finiten Formen sowie Ib - Falscher Gebrauch von finiten Formen.

Bei finiten Verbformen können morphologische Fehler in drei weitere Gruppen aufgeteilt werden. Die erste Gruppe bilden Fehler in der Person- und Numeruskongruenz des finiten Verbs und des Subjekts. Im Gegensatz zum Russischen, in dem das Subjekt und das Prädikat nur im Präsens und Futur (nicht im Präteritum) in Person und Numerus sowie in Numerus und Genus im Präteritum übereinstimmen müssen, ist im Deutschen in allen Tempora die Person- und Numeruskongruenz des finiten Verbs und des Subjekts obligatorisch, während das deutsche Verb die Kategorie des Genus nicht kennt.

Die zweite Untergruppe bilden morphologische Fehler, die aufgrund des falschen Gebrauchs von Tempora auftreten und die dritte Untergruppe bilden Fehler, welche aufgrund des falschen Gebrauchs des Modus' entstanden sind.

Fehlergruppe II betrifft den Verbstamm und beinhaltet Verbformen, die nach einem falschen Muster gebildet wurden und in der Sprache nicht existieren. Gruppe IIa umfasst morphologische Verbfehler, welche die fehlerhafte Bildung von regelmäßigen Verben betrifft. In Gruppe IIb sind Fehler zusammengefasst, welche das falsche Bildungsmuster von unregelmäßigen Verben betreffen. Darunter werden die Präteritumsbildung von unregelmäßigen Verben nach dem Bildungsmuster von regelmäßigen sowie das Weglassen von inneren Flexionen unterschieden. 


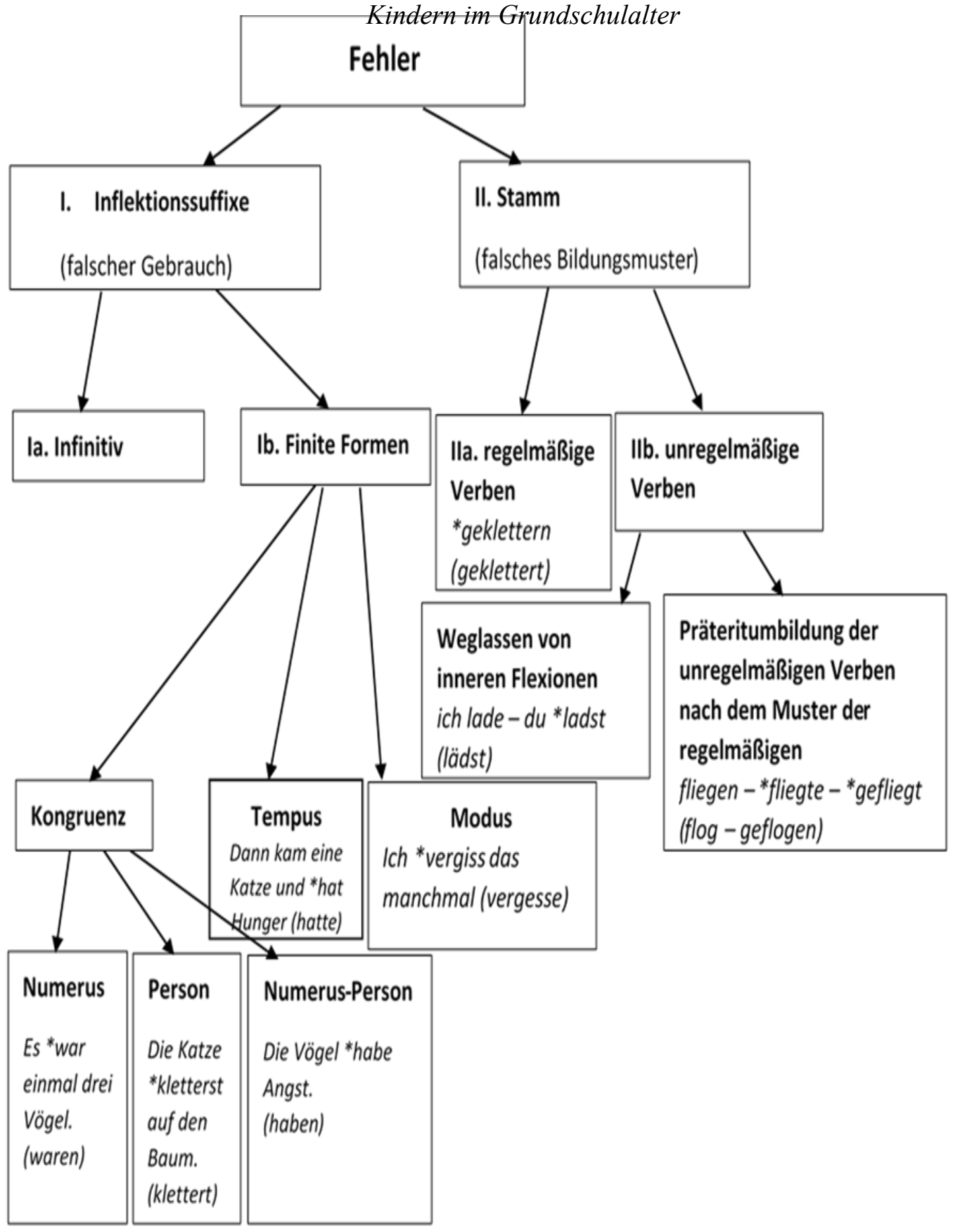

Abbildung 3: Klassifikation der morphologischen Verbfehler im Deutschen

\section{$5 \quad$ Fragestellungen und Hypothesen}

Im Folgenden wird der Gebrauch von Verben in den von russisch-deutsch bilingualen Kindern auf Deutsch und auf Russisch produzierten Narrativen untersucht. Dabei sollen folgende Fragen beantwortet werden: 
- Welche morphologischen Verbfehler machen russisch-deutsch bilinguale Kinder und in welcher Sprache ist die Fehlerzahl größer?

- Welche morphologischen Verbfehler kommen oft vor und sind damit typisch für bilinguale Kinder und welche Fehler sind lernerspezifisch und lassen sich nur bei einzelnen Kindern finden?

- Welche Rolle spielen das Alter und die Kontaktzeit mit der Zielsprache beim Erwerb der morphologischen Kategorien des Verbs im Russischen und im Deutschen?

- Machen simultan bilinguale Kinder mehr Fehler als sukzessiv bilinguale Kinder oder umgekehrt? Wie ist dies erklärbar?

Hypothese I: Da sich generell bei bilingualen Kindern solche Fehler feststellen lassen, welche für die Kindersprache typisch sind (Anstatt 2006:20), wird angenommen, das von den zu untersuchenden Kindern diese auch gemacht werden. Da Gagarina (2011:151) Fehler dokumentiert, die nur für bilinguale Kinder typisch sind (sogenannte unique bilingual errors), wird erwartet, dass neben anderen auch solche bei den an der Studie teilnehmenden Kindern vorkommen werden.

Hypothese II: Da die Umgebungssprache mit dem Alter an Dominanz zunimmt (Kauschke 2012:124), wird angenommen, dass bei jüngeren sukzessiv bilingualen Kindern die wenigsten morphologischen Verbfehler im Russischen auftreten werden, wobei die Fehlerzahl im Russischen bei den gleichaltrigen sukzessiv bilingualen Kindern sowie älteren simultan und sukzessiv bilingualen Kindern größer sein wird. Die Fehlerzahl im Deutschen wird hingegen mit dem Alter sinken.

Sollte diese Annahme zutreffen, wird erwartet, dass IIa) bei den jüngeren simultan bilingualen Kindern (Gruppe A) eine gleiche Fehlerzahl im Russischen und im Deutschen dokumentiert wird; IIb) die jüngeren sukzessiv bilingualen Kinder (Gruppe B) aufgrund der geringeren Kontaktzeit mit dem Deutschen mehr Fehler in den auf Deutsch erzählten Geschichten produzieren werden; IIc) bei den älteren simultan bilinguale Kindern (Gruppe C) hingegen mehr Fehler in den auf Russisch erzählten Geschichten gefunden werden; IId) die Fehlerzahl bei den älteren sukzessiv bilingualen Kindern (Gruppe D) in beiden Sprachen entweder gleich sein wird, oder bei denen aufgrund der bereits langen Kontaktzeit mit dem Deutschen mehr Fehler im Russischen festgestellt wird. 


\section{Probanden und Untersuchungsmethode}

\subsection{Untersuchungsgruppen}

Für diese Untersuchung werden Daten von insgesamt 37 russisch-deutsch bilingualen Kindern analysiert. Davon sind zum Zeitpunkt der Untersuchung 22 Kinder im Alter zwischen 7;11 und 10;5 und 15 Kinder im Alter zwischen 6;9 und 7;5. Innerhalb der Altersgruppen werden die Kinder in simultan und sukzessiv bilingual (s. Kap. 2) unterteilt. Der Kontakt mit dem Russischen beginnt bei allen Kindern mit der Geburt $(0 ; 0)$.

Tabelle 1: Gruppen der simultan bilingualen Kinder

\begin{tabular}{|c|c|c|c|c|c|c|c|}
\hline \multicolumn{4}{|c|}{ Untersuchungsgruppe A } & \multicolumn{4}{|c|}{ Untersuchungsgruppe C } \\
\hline Kind & Alter & $A o O$ & $L o E$ & Kind & Alter & $\mathrm{AoO}$ & LoE \\
\hline s-01 & $6 ; 10$ & $0 ; 10$ & $6 ; 0$ & g001 & $9 ; 1$ & $1 ; 4$ & $7 ; 9$ \\
\hline$s-02$ & $7 ; 5$ & $1 ; 7$ & $5 ; 10$ & g002 & $9 ; 8$ & $0 ; 0$ & $9 ; 8$ \\
\hline$s-04$ & $6 ; 11$ & $0 ; 9$ & $6 ; 2$ & g005 & $9 ; 10$ & $0 ; 0$ & $9 ; 10$ \\
\hline$s-08$ & $7 ; 4$ & $1 ; 9$ & $5 ; 7$ & g006 & $9 ; 4$ & $0 ; 0$ & $9 ; 4$ \\
\hline s-09 & $6 ; 11$ & $1 ; 10$ & $5 ; 1$ & g007 & $8 ; 6$ & $0 ; 0$ & $8 ; 6$ \\
\hline$s-10$ & $6 ; 8$ & $0 ; 9$ & $5 ; 11$ & g008 & $9 ; 4$ & $0 ; 0$ & $9 ; 4$ \\
\hline$s-12$ & $6 ; 5$ & $1 ; 3$ & $6 ; 2$ & g009 & $9 ; 8$ & $0 ; 0$ & $9 ; 8$ \\
\hline$s-15$ & $7 ; 2$ & $1 ; 10$ & $5 ; 4$ & $\mathrm{~g} 010$ & $9 ; 4$ & $0 ; 0$ & $9 ; 4$ \\
\hline & & & & $\mathrm{g} 012$ & $9 ; 5$ & $0 ; 0$ & $9 ; 5$ \\
\hline & & & & g014 & $10 ; 0$ & $1 ; 11$ & $8 ; 1$ \\
\hline & & & & $\mathrm{g} 016$ & $9 ; 7$ & $0 ; 0$ & $9 ; 7$ \\
\hline & & & & g018 & $8 ; 3$ & $0 ; 0$ & $8 ; 3$ \\
\hline & & & & $\mathrm{g} 021$ & $9 ; 1$ & $0 ; 8$ & $8 ; 5$ \\
\hline & & & & g023 & $10 ; 5$ & $0 ; 0$ & $10 ; 5$ \\
\hline
\end{tabular}

Note. AoO (Age of Onset $)=$ Alter beim Erwerbsbeginn, Deutsch; LoE (Length of exposure $)=$ Spracherwerbsdauer, Deutsch.

Tabelle 2: Gruppen der sukzessiv bilingualen Kinder

\begin{tabular}{|l|l|l|l|l|l|l|l|}
\hline \multicolumn{4}{|l|}{ Untersuchungsgruppe B } & \multicolumn{4}{l|}{ Untersuchungsgruppe D } \\
\hline Kind & Alter & AoO & LoE & Kind & Alter & AoO & LoE \\
\hline s-03 & $7 ; 1$ & $2 ; 2$ & $4 ; 11$ & g003 & $9 ; 2$ & $2 ; 6$ & $6 ; 8$ \\
\hline s-05 & $7 ; 0$ & $4 ; 2$ & $2 ; 10$ & g004 & $9 ; 1$ & - & - \\
\hline s-06 & $6 ; 9$ & $4 ; 1$ & $2 ; 8$ & g011 & $9 ; 2$ & $2 ; 0$ & $7 ; 2$ \\
\hline s-07 & $7 ; 0$ & $3 ; 11$ & $3 ; 1$ & g015 & $9 ; 7$ & $4 ; 2$ & $5 ; 5$ \\
\hline s-11 & $7 ; 5$ & $2 ; 6$ & $4 ; 11$ & g017 & $9 ; 5$ & $4 ; 0$ & $5 ; 5$ \\
\hline s-13 & $7 ; 3$ & $2 ; 11$ & $4 ; 4$ & g019 & $8 ; 8$ & $3 ; 0$ & $5 ; 8$ \\
\hline s-14 & $6 ; 11$ & $2 ; 10$ & $4 ; 1$ & g020 & $7 ; 11$ & $2 ; 0$ & $5 ; 11$ \\
\hline
\end{tabular}

Note. AoO (Age of Onset) = Alter beim Erwerbsbeginn, Deutsch; LoE (Length of exposure) $=$ Spracherwerbsdauer, Deutsch. 
Alle untersuchten Kinder besuchen eine staatliche bilinguale Schule in Berlin. Die Schüler werden dort in deutscher und russischer Sprache alphabetisiert, erhalten seit der Einschulung einen regelmäßigen Unterricht in beiden Sprachen, der von Muttersprachlern erteilt wird und sie werden von L1-Sprechern des Deutschen und des Russischen im Hort betreut.

Laut Ergebnissen von IQ-Tests, Angaben der Eltern in den am ZAS entwickelten Fragebögen sowie Gesprächen mit den Klassenlehrern weisen die an der Studie teilnehmenden Kinder keine Evidenz von SLI (umschriebener Sprachentwicklungsstörung) auf. Alle Kinder besuchen reguläre Klassen in der Schule und bekommen gute bis sehr gute Noten. Daraus lässt sich schließen, dass es sich bei den untersuchten Kindern um Kinder mit einer typischen Sprachentwicklung handelt.

\subsection{Methode der Untersuchung}

Von allen Kindern werden zweimal jeweils auf Deutsch und auf Russisch Daten in einem zeitlichen Abstand von 1-2 Wochen erhoben.

Für die Datensammlung werden die Bildergeschichten aus dem Multilingual Assessment Instrument for Narratives (MAIN) (Gagarina et al. 2012), das im Rahmen der internationalen Zusammenarbeit des Projekts COST Action IS0804 Language Impairment in a Multilingual Society: Linguistic Patterns and the Road to Assessment in order to assess narrative production and comprehension skills of children from 3 to 10 years entwickelt wurde, verwendet.

Für die vorliegende Studie werden Geschichten, die im ModellgeschichteFormat (telling after listening to a model story) erzählt wurden, analysiert. Um den monolingualen Kontext während der Testsituation zu fördern, wurde jedes Kind von zwei verschiedenen Testpersonen jeweils in der Muttersprache der Testperson getestet.

Für die Untersuchung werden vier Geschichten verwendet, wobei die Geschichten Cat und Dog als model story dienen und die Geschichten Baby Birds $(B B)$ und Baby Goats $(B G)$ von den Kindern nach dem Muster von Cat and Dog erzählt werden. Die Geschichten sind so ausbalanciert, dass jedes Kind jede Geschichte nur einmal kennenlernt. ${ }^{4}$

4 Erhebungsverfahren vgl. Gagarina et al. 2012. 


\section{$7 \quad$ Fehleranalyse}

\subsection{Morphologische Verbfehler im Russischen}

Die erste große Gruppe bilden morphologische Fehler, die mit einem falschen Gebrauch bereits in der Sprache existierender Verbformen verbunden sind. Das heißt, dass ein Kind eine in der Sprache vorhandene Verbform in einem falschen Kontext verwendet.

Den größten Teil der morphologischen Verbfehler beim Gebrauch der finiten Verbformen bilden die Kongruenzfehler. Am häufigsten treten dabei Genus-Kongruenzfehler im Präteritum auf:

(1) $*_{s}-06$ : Eta spas.

\%ger: Diese rettete.

Daneben treten bei zwei Kindern Numerus-Kongruenzfehler auf, die als „unique-bilingual“ (Gagarina, Armon-Lotem, Gupol 2006:9) gelten:

(2) *g015: no eto zametil m mama i drugoj telenok.

\%ger: na das bemerkte die Mama und das andere Kalb.

(3) $*_{s}-15$ : Segodnja budet u menja na obed tochno kozochki.

\%ger: Heute wird es bei mir zum Mittagessen bestimmt Ziegen geben.

Kongruenzfehler können entstehen, wenn der Genus noch nicht erworben ist oder wenn das Genus eines Substantivs aus einer Sprache in die andere übertragen wird (vgl. Dt.: der Rabe (mask.) = Rus.: vorona (fem.)). Dabei ist anzumerken, dass bei den meisten Kindern neben der falschen Subjekt-Verb-Kongruenz auch richtige Formen auftreten, wie zum Beispiel beim Kind mit der Nummer s-14:

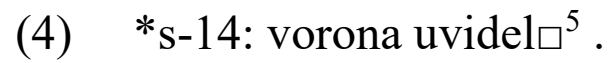

\%ger: Die Krähe sah.

(5) *s-14: vorona ukusila lisu za xvost.

\%ger: Die Krähe biss den Fuchs an den Schwanz.

(6) *s-14: i vorona ispugala lisu.

\%ger: Und die Krähe beängstigste den Fuchs.

Einen weiteren Fehler beim Gebrauch der finiten Verbformen bilden die Aspektfehler. Häufiger gebrauchten die untersuchten Kinder imperfektive Verben

$5 \quad$ statt uvidela 
anstelle perfektiver (Beispiele 7 -9), aber auch perfektive anstelle imperfektiver (Beispiel 10):

(7) *s-09: i potom letela ${ }^{6}$ ptichka.

\%ger: und dann flog das Vögelchen.

(8) *g001:i lisica na nego napadala ${ }^{7}$. \%ger: und der Fuchs griff ihn an.

(9) *g004: i xotela malen'kogo kozlenochka est ${ }^{\text {r8 }}$. \%ger: und wollte das kleine Zieglein essen.

(10) *g003: i nikogda bol'she tuda ne pribezhala ${ }^{9}$. \%ger: und niemals mehr kam hierher zurück.

Bezüglich des Tempus-Gebrauchs lässt sich im Russischen nur ein Fehler dokumentieren:

(11) *g019: a koshka zalezaet i zaxvatila pochti odnogo ptenchika.

\%ger: und die Katze klettert hoch und hat schon fast das eine Vöglein gefangen.

Korrekt wäre in diesem Satz die Präteritumsform vom Verb zalezat' (zalezala, zu Dt.: kletterte, pf.) zu verwenden, da das zweite Prädikat des Satzes (zachvatila, zu Dt.: gefangen nahm, pf.) im Präteritum steht sowie die ganze Geschichte im Präteritum erzählt wurde.

Bei einigen Kindern lassen sich Infinitivformen anstelle flektierter Formen dokumentieren:

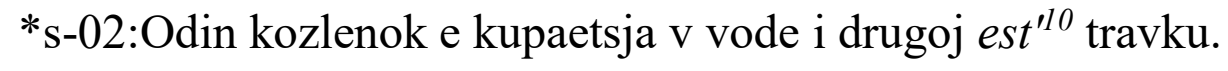

\%ger: Ein Zicklein badet im Wasser und das Andere essen Graß.

Die zweite große Fehlergruppe bilden die innovativen Verbformen, die in der Standardsprache nicht existieren und von den Kindern nach einem falschen Muster gebildet wurden. Diese Fehlergruppe wird in zwei weitere Untergruppen unterteilt.

In Gruppe IIa konnte bei den zu untersuchenden Kindern kein Fehler dokumentiert werden. In Gruppe IIb kamen alle drei Fehlertypen vor: Einfluss der Verbklasse, Einfluss des „Partner“-Stammes sowie Stammerweiterung durch

\footnotetext{
6 statt priletela

7 statt napala

8 statt $s^{\prime}$ est'

9 statt pribegala

10 statt est
} 
Reflexivsuffix. Ein Fehler, der aufgrund des Einflusses des „Partner“-Stammes entstanden ist, lässt sich nur einmal bei Kind s-09 dokumentieren:

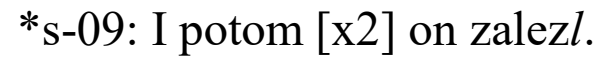

\%ger: Und dann kletterte er hoch.

Offenbar kennt das Kind die Regel, dass das Präteritum im Russischen durch das Anhängen des Suffixes $-l$ an den Infinitivstamm gebildet wird. Bei maskulinen Verbformen, deren Stamm auf b, p, g, k, ch, z, s, r endet, wird im Präteritum kein Suffix - $l$ angehängt (Švedova 2005:628). Die korrekte Präteritumsform des Verbs zalezt' (zu Dt.: hochklettern, pf.) in der 3. Person Singular, mask. lautet daher zalez, wobei die feminine Präteritumsform in der 3. Person Singular wiederum das Suffix - $l$ enthält und zalezla lautet.

Ein Beispiel für die falsche Infinitivform findet sich bei Kind g021:

*g021: na vtoroj kartinke ja vizhu, chto mama pomogaet kozliku iz vody vylezt'.

\%ger: auf dem zweiten Bild sehe ich, dass die Mama dem kleinen Ziegenbock hilft, aus dem Wasser herauszukommen.

Dabei hat das Kind ein falsches Infinitivbildungsmuster gewählt und gebraucht die Form *vylezt', analog zum Verb mit derselben Wurzel zalezt' (zu Dt.: hochklettern, pf.). In der russischen Standardsprache existiert die Infinitivform vylezti (zu Dt.: herausklettern, pf.). Bei einigen Autoren wird jedoch die Form vylezt' als umgangssprachlich bzw. veraltet bezeichnet (vgl. Ožegov/Švedova 1997:114). Einen ähnlichen Fehler macht Kind g004 und bildet eine falsche Infinitivform vom Verb vynut' (zu Dt.: herausnehmen, pf.):

*g004: i potom on, m, on nu, papa xotel ego vyn'ti.

\%ger: und dann er, na, der Papa wollte ihn herausnehmen

Das Kind *s-09 erweitert den Stamm und gebraucht anstelle von der Infinitivform zalezt' (zu Dt.: hochklettern, pf.) die Form *zalezit' nach dem Muster des Verbs zalepit' (zu Dt.: zukleben, pf):

(16) *s-09: Koshka xotela zalezit'.

\%ger: Die Katze wollte hochklettern

Es ließen sich auch einige Fehlerbeispiele mit Stammerweiterung durch Suffixe dokumentieren: 
(17) *s-02: I kozljonka raduetsja, chto [x2] ptica ukus ukusala $\mathrm{v}$ hvost lisy \%ger: Und das Zicklein freut sich, dass der Vogel den Fuchs am Schwanz gebissen hat

(18) *g004: tam upadal malen'kij kozlenok kozlenochek v vodu. \%ger: Dort fiel ein kleines Zicklein ins Wasser.

(19) *g020: a drugoj kozlenok edaet travu. \%ger: und das andere Zicklein ißt Graß.

Alle diese Beispiele sind nach dem Muster der sogenannten „Partnerverben“ gebildet. Das Präteritum vom perfektiven Verb ukusit' (zu Dt: beißen, pf.) im Beispiel (17) wird nach dem Muster seines imperfektiven Partners kusat' gebildet. Möglich ist auch, dass das Kind die richtige Infinitivform des Verbs nicht kennt und davon ausgeht, dass dieses Verb im Infinitiv *ukusat' heißt und regelmäßig durch das Anhängen vom Suffix $-l$ die Präteritumsform bildet. Das gleiche Fehlermuster ist auch beobachtbar an Beispiel (18). Das Aspektpaar ist padat'upast' (zu Dt.: hinfallen bzw. herunterfallen) und entweder übernimmt das Kind das Bildungsmuster des imperfektiven Verbpartners, oder geht von einer falschen Infinitivform (*upadat'), die durch Präfigierung vom Kind gebildet wird, aus.

Beispiel (19) stellt die Bildung der 3. Person Sg. vom Verb est' (zu Dt.: essen, ipf.) analog zum Verb obedat' (zu Dt.: Mittag essen, ipf.) (obedat'- obedaet vgl. est ${ }^{*}$ *edaet) dar. Die korrekte Form in der 3. Person Sg. lautet est.

Im nächsten Beispiel wird das Verb radovat'sja (zu Dt.: sich freuen, ipf.) vom Kind nach einem falschen Muster konjugiert:

*s-10: I kozljonka radovaetsja.

\%ger: Und das Zicklein freut sich.

Die Verben mit dem Suffix -ova- im Infinitiv „,verlieren“ bei der Konjugation im Präsens dieses Suffix und bekommen das Suffix $-u$ - und die Personenendung (vgl. Švedova 2005:650f). Die korrekte Form muss daher radujetsja lauten. Das Kind bildet die Präsensform der 3. Person Singular des Verbs radovat'sja nach dem Konjugationsmuster des Verbs katat'sja (zu Dt.: fahren, ipf.): (on) kataetsja. Einige Kinder erweiterten den Verbstamm durch das Reflexivsuffix und bildeten dadurch eine in der Sprache nicht existierende Verbform:

${ }^{*}$ s-12: Nu sobaka tozhe ne xotelas $^{11}$ povernut'sja.

\%ger: Und der Hund wollte sich auch nicht umkehren.

${ }^{11}$ statt xotela 
*g010: pes rasserdilsja i dognalsja ${ }^{12}$ za kot koshkoj. \%ger: Der Hund ist böse geworden und jagte der Katze hinterher.

Die korrekte Form im Beispiel (21) wäre ohne das Reflexivsuffix -sja-xotela (zu Dt.: wollte, ipf.). Das Verb *dognat'sja wird analog zum Verb pognat'sja (zu Dt.: hinterherjagen, pf.) gebildet. In der russischen Standardsprache existiert jedoch nur das Verb dognat' (zu Dt.: einholen, pf.).

Bei einigen Kindern kommen morphologische Verbfehler vor, die sich nicht eindeutig zuordnen lassen, da nicht festgestellt werden kann, aus welchem Grund der Fehler entstanden ist, bzw. die Semantik des Verbes unklar ist. Kind s03 produziert die Verbform * ${ }^{*}$ stalis', die im Russischen nicht existiert:

*s-03: A mama i s kozlenkami vdvoem vmeste teper'. Tam stalis'.

\%ger: Und die Mama mit den Zieglein sind nun zuzweit. Dort geblieben.

Das Reflexivverb *stat'sja gibt es im Russischen nicht, das Verb stat' (zu Dt.: werden) wäre in diesem Kontext semantisch falsch, so müsste die korrekte Form im Beispiel (23) stojali (zu Dt.: standen, ipf.) bzw. ostalis' (zu Dt.: geblieben sind, pf.) lauten.

Bei Kind s-01 ist die Form *akusi dokumentiert, wobei die korrekte Form ukusila (zu Dt.: biss, pf.) lauten müsste:

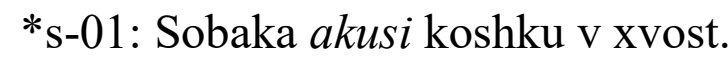

\%ger: Der Hund biss die Katze in den Schwanz.

Der perfektive Aspekt des Verbs kusat' (zu Dt.: beißen, ipf.) wird durch das Anhängen von eines falschen Präfixes $a$ - anstelle des Präfixes $u$ - gebildet. Außerdem wird das Verb falsch konjugiert und bekommt die Endung - $i$, die den Imperativ Singular ausdrückt. Für Indikativ Singular Femininum Präteritum ist das Suffix - $l$ - sowie die Endung -a obligatorisch.

Kind g004 bildet die Präteritumsform des Verbs spasti (zu Dt.: retten, pf.) falsch und ersetzt den Stammvokal - $a$ - durch -o-:

*g004: On spos malen'kogo.

\%ger: Er hat den Kleinen gerettet.

Kind g001 bildet durch das Anhängen einer falschen Endung an den Stamm eine fehlerhafte Verbform:

${ }^{12}$ statt dognal koshku oder pognalsja za koshkoj 
*g001: \&nu, mama pomogalu malen'komu.

\%ger: na, die Mama half dem kleinen

Das Kind gebraucht den richtigen Verbstamm im Präteritum und hängt anstelle der einzig möglichen Endung - $a$ für Femininum im Präteritum die Endung $-u$ an. Dadurch wird eine Form gebildet, die in der Standardsprache nicht existiert und damit eine Innovation ist.

Bei Kind s-06 kommt eine Verbform mit einer unklaren semantischen Bedeutung vor:

*s-06: I togda ptic lisa ubezhala. I ptichka zanjobyla.

\%ger: Und dann rannte der Fuchs weg. Und der Vogel unclear.

Die Form *zanjobyla weist auf morphologische Merkmale eines Verbes im Indikativ Präteritum Singular Femininum auf, lässt sich aber semantisch nicht deuten. Womöglich handelt es sich dabei um drei Wörter $z a{ }^{*}$ njo byla = za nej poletela (zu Dt.: flog ihr hinterher). Die genaue Semantik dieser Aussage kann jedoch nicht festgestellt werden.

\subsection{Morphologische Verbfehler im Deutschen}

Im Deutschen lassen sich deutlich weniger morphologische Verbfehler dokumentieren. Diese Fehler werden wie im Russischen in zwei größere Gruppen mit Unterteilung in Untergruppen eingeteilt.

Die erste größere Gruppe bilden solche Fehler, die den Verbgebrauch betreffen. Darunter lassen sich Tempus-, Modus- und Kongruenzfehler dokumentieren. Andere morphologische Verbkategorien sind bei den untersuchten Kindern nicht von Fehlern betroffen. Falscher Gebrauch von Tempusformen kommt nur bei einem Kind vor:

(28) *g005: Dann kam ein ne Katze oder ein Kater und hat hm riesen Hunger.

(29) *g005: Und dann ha hat der Hund dann is [: ist] der Hund gekommen und hat hm den Schwanz von den Kater genommen und runter also gedrueckt, dass der Kater runterfällt.

Bei drei Kindern sind Kongruenzfehler dokumentiert:

*s-06: Es war einmal drei Vögel. 
(31) *s-03: Kleine Vogel ${ }^{13}$ möchte essen.

(32) *g018: Also, es lebten einmal eine Vogelfamilie und die, also die kleinen Kinder, die hatten Hunger. *g018: Und dann sind die Katze gekommen und die Katze wollte die [x 2] kleinen Kinder \&essen xx essen.

Bei allen Beispielen $(30$ - 33) handelt es sich um die Numerus-Kongruenz zwischen dem Subjekt und Prädikat im Präteritum. Bei zwei Kindern sind Modusfehler dokumentiert, bei denen es sich um den Gebrauch von einer Imperativform anstelle von einer Indikativform handelt:

(34) *s-04: Ich vergiss das manchmal.

(36) *s-04: Ich vergiss immer die Namen!

(37) *g020: Er wollte einen kleinen Vogel also mitnehmen nach unten und dann auffriss.

Die weitere größere Gruppe bilden Wortformen, die in der deutschen Standardsprache nicht existieren, aber aus in der Sprache existierenden Morphemen zusammengesetzt sind. Bei den untersuchten Kindern ist ausschließlich die Präteritumsbildung betroffen, wobei lediglich ein Fehler bei der Perfektbildung eines regelmäßigen Verbs vorkommt:

$$
\text { *g009: Er ist auf den Baum geklettern }{ }^{14}
$$

Das Kind bildet das Perfekt des regelmäßigen Verbs klettern nach dem Muster der unregelmäßigen.

Bei der Präteritumsbildung der unregelmäßigen Verben treten hingegen die meisten Fehler auf:

(39) *s-06: Die Vogelmama fliegte ${ }^{15}$ mal weg.

(40) *s-08: Da kommte ${ }^{16}$ ein Fuchs vorbei.

(41) *s-10: Dann hat der Hund von der Katze den Schwanz gebei $\beta t^{17}$.

Diese Fehlerart stellt eine Übergeneralisierung dar und zeigt, dass ein grammatisches Muster erworben wurde, aber noch nicht ausdifferenziert

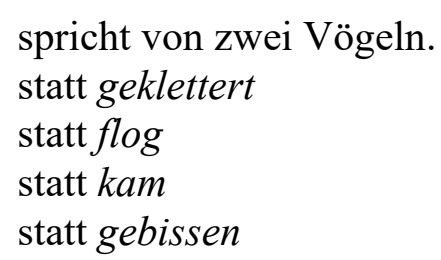


angewendet wird. Bei monolingualen Kindern lässt sich dieser Fehler auch nach dem Alter von 3;0 noch lange beobachten (Kauschke 2012:82). Bei bilingualen Kindern erstreckt sich der Erwerb von Partizipien über eine längere Zeitperiode. Anzumerken ist hier auch, dass dieser Fehler nur bei den jüngeren Kindern aus den Gruppen A und B dokumentiert wurde und bei den älteren (Gruppen C und D) gar nicht vorkommt. Dabei gebrauchen die jüngeren Kinder neben den falschen Formen der unregelmäßigen Verben auch die richtigen. Auch Kind s-08, bei dem die meisten Fehler dieser Art dokumentiert werden konnten, bildet das Präteritum des Verbs denken einmal falsch, einmal richtig:

*s-08: denkte der Vogel.

(43) *s-08: Da dachte der Vogel.

\subsection{Zusammenfassung}

Bei der Analyse der morphologischen Verbfehler stellte sich heraus, dass die Fehlerzahl im Deutschen deutlich niedriger als im Russischen ist und mit zunehmendem Alter sinkt. 50\% der Kinder aus Gruppe A sowie 43\% der Kinder aus Gruppe B machen keine morphologischen Verbfehler im Deutschen. Die Fehleranzahl im Deutschen liegt bei den anderen Kindern dieser beiden Gruppen bei ein bis zwei. Eine einzige Ausnahme ist dabei das simultan bilinguale Kind s08 aus Gruppe A, welches insgesamt zehn morphologische Verbfehler macht, die jedoch alle zu einer Fehlerart gehören und die Präteritumsbildung von unregelmäßigen Verben betreffen.

Bei $80 \%$ der Kinder aus Gruppe C sowie bei 86\% der Kinder aus Gruppe D kommen keine morphologischen Verbfehler im Deutschen vor. Bei den Kindern dieser beiden Gruppen, bei denen morphologische Fehler dokumentiert wurden, beträgt die Fehlerzahl maximal zwei.

Im Russischen haben die untersuchten Kinder mehr Fehler produziert. In Gruppe A wurden bei 25\%, in Gruppe B bei $43 \%$, in Gruppe C bei 53\% sowie in Gruppe D bei $43 \%$ Kindern keine morphologischen Verbfehler in den auf Russisch erzählten Geschichten dokumentiert.

Daraus folgt, dass die Prozentzahl der sukzessiv bilingualen Kinder der Gruppe B, welche keine morphologischen Verbfehler machen, sowohl im Russischen, als auch im Deutschen gleich ist (43\%). In allen anderen Gruppen (A, $\mathrm{C}$ und D) ist die Prozentzahl der Kinder, welche keinem morphologischen Verbfehler in den auf Deutsch erzählten Geschichten machen, größer. Der größte Prozentsatz an Kindern, die keinen morphologischen Verbfehler produzieren, ist in der Gruppe der älteren sukzessiv bilingualen Kinder zu finden (Gruppe D, $86 \%)$. 
In allen vier Gruppen gibt es Kinder, die mindestens in einer Sprache keine Fehler machten, manchmal produzierten aber diese Kinder kurze Geschichten, bei denen die Verbanzahl eher niedrig war. Dieses Phänomen wurde bereits bei Protassova (2011:290) beschrieben. Kind g012 erzählte auf Russisch eine lange Geschichte, gebrauchte jedoch wenige Verben und von insgesamt 18 Aussagen brach es sieben ab, sodass die Geschichte insgesamt als zusammenhangslos und unverständlich erschien, jedoch keinen morphologischen Verbfehler enthielt.

Einige Kinder produzierten lange Geschichten und machten zudem keine morphologischen Verbfehler.

Die Fehlerquote, d.h. der Prozentsatz an Verben mit morphologischen Fehlern von der Gesamtzahl der Verbtokens liegt in den auf Russisch erzählten Geschichten bei den Kindern aus Gruppe A bei 5,6\%, bei den Kindern aus Gruppe B bei 7\%, bei den Kindern aus Gruppe C bei 4\% und bei den Kindern aus Gruppe D bei 7,8\%.

Dabei zeigten die besten Ergebnisse im Russischen ältere simultan bilinguale Kinder aus Gruppe C. Im Deutschen liegt die Fehlerquote bei den Kindern aus Gruppe A bei 9\%, bei den Kindern aus Gruppe B bei 6\%, bei den Kindern aus Gruppe $\mathrm{C}$ bei 1,8\% sowie bei den Kindern aus Gruppe D bei 0,9\%. Dabei zeigten die besten Ergebnisse im Deutschen ältere sukzessiv bilinguale Kinder aus der Gruppe D.

Während einige morphologische Verbfehler bei mehreren Kindern vorkamen, konnten andere nur bei einzelnen Kindern dokumentiert werden. Im Rahmen dieser Studie wird davon ausgegangen, dass alle Fehler, die drei oder mehr Kinder produziert haben, als typische Fehler für bilinguale Kinder bewertet werden können. Morphologische Verbfehler, die bei einem bis zwei Kindern zu finden sind, gelten als lernerspezifisch und individuell. $\mathrm{Zu}$ den häufigsten Fehlern in der Kategorie falscher Gebrauch der Verbformen im Russischen gehören Kongruenzfehler, die von acht Kindern gemacht wurden und Aspektfehler, die vier Kinder produzierten. Außerdem wurden von vier Kindern Infinitivformen anstelle einer flektierten Form gebraucht.

Nach einem falschen Bildungsmuster wurden von fünf Kindern Fehler unter dem Einfluss der Verbklasse produziert, von zwei Kindern wurden falsche Verbformen mit der Stammerweiterung durch das Reflexivsuffix gebildet und von einem Kind wurde eine fehlerhafte Form unter dem Einfluss des Partnerstammes gebildet.

Im Deutschen treten in der Kategorie falscher Gebrauch bei drei Kindern Kongruenzfehler auf, zwei Kinder gebrauchen den Modus falsch und bei einem Kind wurde ein Tempusfehler dokumentiert. In der Fehleruntergruppe falsches Bildungsmuster bilden sieben Kinder Präteritumsformen unregelmäßiger Verben 
nach dem Muster der regelmäßigen und nur bei einem Kind wurde ein regelmäßiges Verb nach dem Muster der unregelmäßigen gebildet.

$\mathrm{Zu}$ den typischen morphologischen Verbfehlern bei russisch-deutsch bilingualen Kindern im Grundschulalter zählen somit im Russischen:

- Kongruenzfehler

- Infinitivgebrauch anstelle von einer flektierten Form

- Gebrauch von einem Verb des falschen Aspekts

- Falsche Formbildung unter dem Einfluss der Verbklasse.

Bei den Kongruenzfehlern handelt es sich dabei bei den meisten Kindern um die Genus-Kongruenz. Dieser Fehler wurde auch bei russisch-hebräisch bilingualen Kindern beobachtet (Gagarina, Armon-Lotem, Gupol 2006:9). Wie in Kapitel 3 dargestellt, nimmt der Genus-Erwerb bei bilingualen Kindern unterschiedlich viel Zeit in Anspruch und manche Kinder beherrschen das Genussystem des Russischen erst nach dem 10. Lebensjahr oder noch später (vgl. Dieser 2009:166). Auch der Numeruskongruenz-Fehler im Futur, der unique bilingual ist und bei russisch-hebräisch bilingualen Kindern dokumentiert wurde (Gagarina, ArmonLotem, Gupol 2006:9), kam in der vorliegenden Studie bei zwei Kindern vor. Der Gebrauch von Infinitivformen anstelle einer flektierten Form kann darauf zurückgeführt werden, dass der Infinitiv die einzige erworbene Form eines konkreten Verbs ist und das Kind nicht weiß, wie es konjugiert wird. Man könnte auch einen Zusammenhang mit dem Synkretismus der Verbformen im Deutschen annehmen, den das Kind auch in die russische Sprache überträgt.

Falscher Gebrauch von Aspektpartnern ist ein Fehler, der sehr häufig bei bilingualen Kindern dokumentiert wird und womöglich damit zusammenhängt, dass das Deutsche diese grammatische Form nicht kennt und ein Kind das Verb direkt vom Deutschen ins Russisch ,übersetzt“, ohne auf den Aspekt zu achten. Falsche Formbildung unter dem Einfluss einer Verbklasse stellt eine Übergeneralisierung dar und zeigt, dass ein Verbbildungsmuster erworben ist, aber noch nicht ausdifferenziert angewendet werden kann.

Im Deutschen kamen bei den Kindern folgende Fehlertypen am häufigsten vor:

- Kongruenzfehler

- Bildung der Präteritumsformen der unregelmäßigen Verben nach dem Muster der regelmäßigen.

Alle Kongruenzfehler betreffen die Numerus-Kongruenz zwischen Subjekt und Prädikat und deuten darauf hin, dass die Kinder noch nicht ausdifferenziert Verbflexionen im Singular und im Plural anwenden können. 
Die Bildung der Präteritumsformen der unregelmäßigen Verben nach dem Muster der regelmäßigen stellt eine Übergeneralisierung dar und zeigt, dass ein Bildungsmuster erworben wurde, aber noch nicht ausdifferenziert angewendet wird. Diese Fehlerart wurde auch häufig bei monolingualen Kindern dokumentiert (vgl. Kauschke 2012:82).

\section{$8 \quad$ Fazit und Diskussion}

Der Erwerb der grammatischen Kategorien des Verbs stellt für bilinguale Kinder sowohl im Russischen als auch im Deutschen eine Herausforderung dar. In der vorliegenden Studie wurden morphologische Verbfehler in elizitierten Narrativen bei russisch-deutsch bilingualen Kindern in zwei Altersgruppen sowie mit zwei Erwerbstypen (simultan und sukzessiv) untersucht.

Es wurde festgestellt, dass die meisten Fehler, welche bilinguale Kinder machen, auch bei monolingualen Kindern vorkommen. Es wurden ebenfalls die sogenannten unique bilingual errors bei den an der Studie beteiligten Kindern dokumentiert. Damit bestätigt sich Hypothese I, wonach bei bilingualen Kindern auch solche Fehler auftreten, welche für die Kindersprache als solche typisch sind. Alle Fehler, die in den untersuchten Narrativen vorkamen, wurden klassifiziert, wobei für das Russische die Fehlerklassifikation für monolinguale Kinder von Gagarina (2008) erweitert wurde, um sie aufgrund von den unique bilingual errors auf bilinguale Kinder anwenden zu können.

Morphologische Verbfehler im Deutschen wurden nach der eigenen Klassifikation, die im Kapitel 4.2 vorgeschlagen wurde, klassifiziert.

Für beide Sprachen wurden unter den dokumentierten Fehlern typische morphologische Verbfehler, die bei drei und mehr Kindern vorkamen, sowie lernerspezifische Fehler, die nur bei einzelnen Kindern dokumentiert wurden, ausdifferenziert.

Die Analyse der morphologischen Verbfehler zeigte, dass die Fehlerzahl im Deutschen größer als im Russischen ist und mit zunehmendem Alter sinkt. Damit bestätigt sich die Annahme, dass die Umgebungssprache (im Untersuchungsfall das Deutsche) mit dem Alter an Dominanz zunimmt. Die Ergebnisse bestätigen jedoch nicht, dass die Fehleranzahl im Russischen bei den sukzessiv bilingualen Kindern am niedrigsten ist.

In Hypothese IIa wurde angenommen, dass bei den jüngeren simultan bilingualen Kindern aus Gruppe A die Fehlerzahl im Russischen und im Deutschen gleich sein wird. Diese Kinder zeigten jedoch ein besseres Ergebnis im Russischen, da nur 5,6\% der Verbtokens morphologische Verbfehler enthalten. Im Deutschen liegt die Prozentzahl der Verbtokens mit morphologischen Verbfehlern bei diesen Kindern bei $9 \%$. 
Die in Hypothese IIb beschriebene Annahme, dass die kleineren sukzessiv bilinguale Kinder (Gruppe B) aufgrund der geringeren Kontaktzeit mit dem Deutschen mehr Fehler in dieser Sprache machen würden, bestätigte sich ebenfalls nicht. Im Russischen sind bei diesen Kindern 7\% Verbtokens von morphologischen Verbfehlern betroffen, im Deutschen hingegen nur 6\%.

Es hat sich bestätigt, dass, wie in Hypothese IIc angenommen, bei den älteren simultan bilingualen Kindern (Gruppe C) mehr Fehler in den auf Russisch erzählten Geschichten dokumentiert werden, da das Deutsche als Umgebungssprache bereits an Dominanz zugenommen hat. Die Kinder aus dieser Gruppe produzierten nur 1,8\% Verbtokens mit morphologischen Verbfehlern im Deutschen, aber auch nur 4\% der Verbtokens mit morphologischen Verbfehlern im Russischen, und zeigten damit das beste Ergebnis im Russischen.

In Hypothese IId wurde angenommen, dass die Fehlerzahl bei den älteren sukzessiv bilingualen Kindern (Gruppe D) in beiden Sprachen gleich sein wird, oder sie weniger Fehler im Deutschen produzieren werden. Dies bestätigte sich und außerdem zeigten diese Kinder das beste Ergebnis mit nur 0,9\% der Verbtokens mit morphologischen Verbfehlern im Deutschen. Im Russischen hingegen waren bei den Kindern aus dieser Gruppe 7,8\% Verbtokens von morphologischen Verbfehlern betroffen. Damit ist die Fehlerzahl im Russischen bei den Kindern aus Gruppe D am größten.

Sukzessiv bilinguale Kinder aus den Gruppen B und D zeigten im Deutschen bessere Ergebnisse als die gleichaltrigen simultan bilingualen Kinder aus den Gruppen A und C, bei denen die Kontaktzeit mit dem Deutschen länger war. Anders als in der Studie von Anstatt (2006:22), in der bei simultan bilingualen Kindern der Einfluss der Umgebungssprache größer war und sie mehr Fehler im Russischen machten, wurden bei den simultan bilingualen Kindern der vorliegenden Studie aus den Gruppen A und C weniger Fehler im Russischen dokumentiert, als bei den sukzessiv bilingualen Kindern aus den Gruppen B und D. Die älteren simultan bilingualen Kinder produzierten die wenigsten Fehler im Russischen und zeigten somit im Russischen das beste Ergebnis.

Aber auch innerhalb jeder einzelnen Gruppe sind die Ergebnisse durch eine große Heterogenität gekennzeichnet - während einige Kinder gar keine morphologischen Verbfehler produziert haben, wurden bei den anderen viele morphologische Verbfehler dokumentiert. Dies entspricht den Befunden von Anstatt (2008c:71f u. 2009:111).

Nur bei rund $18 \%$ der an dieser Studie beteiligten Kinder wurden in der deutschen Sprache mehr morphologische Verbfehler als in der russischen dokumentiert. Darunter ist nur ein Kind aus Gruppe C, das mehr morphologische Verbfehler im Deutschen als im Russischen machte.

Das frühe Einstiegsalter sorgt für eine gute Ausgangsposition beim L2Erwerb. Damit sich aber der Spracherwerb erfolgreich vollzieht, sind weitere 
Faktoren wie z.B. die Motivation des Kindes und die Einstellung zu den zu erwerbenden Sprachen wichtig. Diese Faktoren können von Kind zu Kind variieren, was eine mögliche Erklärung für die Heterogenität in den Ergebnissen bei Kindern innerhalb jeder einzelnen Untersuchungsgruppe ist.

\section{References}

Anstatt, Tanja (2006): Leksičeskije i grammatičeskije osobennosti russkoj reči detej-bilingvov v Germanii (na primere rasskazov v kartinkach). In: Cejtlin, Stella N.: Ontolingvistika. Nekotoryje itogi i perspektivy, S.-Petersburg: Zlatoust, pp 19-25.

Anstatt, Tanja/Dieser, Elena (2007): Sprachmischung und Sprachtrennung bei zweisprachigen Kindern (am Beispiel des russisch-deutschen Spracherwerbs). In: Anstatt, Tanja: Mehrsprachigkeit bei Kindern und Erwachsenen. Erwerb, Formen, Förderung, Tübingen: Attempo Verlag, pp 139-162.

Anstatt, Tanja (2008a): Aspektfehler im Russischen mono- und bilingualer Kinder. In: Kempgen, Sebastian et al. (Hrsg.): Deutsche Beiträge zum 14. Internationalen Slavistenkongress Ohrid 2008, München: Otto Sagner.

Anstatt, Tanja (2008b): Aspect and tense in storytelling by Russian, German and bilingual children. In: Russian Linguistics 32, pp 1-26.

Anstatt, Tanja (2008c): Russisch in Deutschland: Entwicklungsperspektiven. Bulletin der deutschen Slavistik 14, pp $67-74$.

Anstatt, Tanja (2009): Der Erwerb der Familiensprache: Zur Entwicklung des Russischen bei bilingualen Kindern in Deutschland. In: Gogolin, Ingrid: Streitfall Zweitsprachigkeit The Bilingualism Controversy, Wiesbaden: VS Verlag für Sozialwesen, pp 111-131.

Böttger, Katharina (2008): Die häufigsten Fehler russischsprachiger Deutschlerner: Ein Handbuch für Lehrende, Münster: Waxmann.

Chilla, Solveig (2013): Kindliche Mehrsprachigkeit: Grundlagen - Störungen - Diagnostik, München: Reinhardt.

Cejtlin, Stella N. (2009): Očerki po slovoobrazovaniju i formoobrazovaniju v detskoj reči. Moskau: Znak.

Cejtlin, Stella N. (2011): Put' v jazyk: odnojazyčie i dvujazyčie. Moskau: Jazyky slav'anskoj kul'tury.

Cejtlin, Stella N. (2013): Rečevye ošibki i ich predupreždenie: učebnoe posobie. Moskau: Librokom.

De Houwer, Annick (1990): The acquisition of two languages from birth: A case study. Cambridge: Cambridge University Press.

Dieser, Elena (2009): Genuserwerb im Russischen und Deutschen: Korpusgestützte Studie zu ein- und zweisprachigen Kindern und Erwachsenen. München: Sagner. 


\section{Olga Vorobyeva}

Gagarina, Natalia (2003): The early verb development and demarcation of stages in three Russian-speaking children. In: Bittner, Dagmar et al.: Development of verb inflection in first language acquisition. A cross-linguistic perspective, Berlin: de Gruyter, pp 131169.

Gagarina, Natalia, Armon-Lotem, Sharon, Gupol, Olga (2006): Developmental variation in the acquisition of L1 Russian verb inflection by monolinguals and bilinguals // BUCLD 31 Proceedings Supplement. Caunt-Nulton H., Kulatilake S., Woo I.-H. (eds.). Boston University, 2006, pp 1-11.

Gagarina, Natalia (2008): Stanovlenije grammatičeskich kategorij russkogo glagola v detskoj reči, Sankt-Petersburg: Nauka.

Gagarina, Natalia (2011): Acquisition and loss of L1 in a Russian-German bilingual child: A case study. In: Cejtlin, Stella N.: Put' v jazyk: Odnojazyčije i dvujazyčije, Moskau: Jazyki slavyanskich kultur, pp 137-163.

Gagarina, Natalia et al. (2012): MAIN: Multilingual Assessment Instrument for Narratives. In: ZASPiL Nr. 56, 2012, pp 1-161.

Gagarina, Natalia (2013): Sprachdiagnostik in der Erstsprache mehrsprachiger Kinder (am Beispiel des Russischen). In: Sprache. Stimme. Gehör; 37, pp 196-200.

Genesee, Fred (1989): Early bilingual development: One language or two? In: Journal of Child language, Vol. 16(1), pp 161-179.

Helbig, Gerhard/Buscha, Joachim (2001): Deutsche Grammatik. Ein Handbuch für den Ausländerunterricht, Berlin: Langenscheidt.

Kauschke, Christina (2012): Kindlicher Spracherwerb im Deutschen. Verläufe, Forschungsmethoden, Erklärungsansätze. Berlin: de Gruyter.

Kreppel, Nicole (2006): Auffälligkeiten im Spracherwerb bilingualer Kinder: Theoretische Grundlage zu Diagnose und Therapie, Wettenberg: Herrmann, J-und-J-Verl.

McLaughlin, Berry (1984): Second-Language Aquisition in Childchood: Volume 1. Preschool Children Second Edition, New Jersey: Lawrence Erlbaum Associates.

Meisel, Jürgen M. (2007): Mehrsprachigkeit in der früheren Kindheit: Zur Rolle des Alters bei Erwerbsbeginn. In: Anstatt, Tanja: Mehrsprachigkeit bei Kindern und Erwachsenen. Erwerb, Formen, Förderung, Tübingen: Attempo Verlag, pp 93-113.

Montrul, Silvina A. (2008): Incomplete Acquisition in Bilingualism. Re-examining the Age Factor, Amsterdam: John Benjamins Publishing Company.

Ožegov, Sergej/Švedova, Natalija (1997): Tolkovyj slovar' russkogo jazyka, Moskau: Az.

Panova, Galina (2010): Morfologija russkogo jazyka: Enciklopedicheskij slovar'-spravochnik, Moskau: KomKniga.

Paradis, Johanne/Genesee, Fred/Crago Martha B. (2011): Dual language development and disorders. A Handbook on Bilingualism and Second Language Learning, 2. Auflage, Brookes: Baltimore. 
Protassova, Ekaterina Ju. et al. (2011): Opisanije kartinok finnsko-russkimi bilingvami. In: Cejtlin, Stella N.: Put' v jazyk: Odnojazyčije i dvujazyčije, Moskau: Jazyki slavjanskich kultur, pp 282-293.

Ramge, Hans (1980): Fehler und Korrektur im Spracherwerb. In: Cherubim, Dieter: Fehlerlinguistik. Beiträge zum Problem der sprachlichen Abweichung, Tübingen: Niemeyer, pp 1-22.

Romaine, Suzanne (1999): Bilingual language development. In: Barrett, Martyn: The development of language, Hove: Psychology Press, pp 251-275.

Ruberg, Tobias (2013): Problembereiche im kindlichen Zweitspracherwerb. In: Sprache. Stimme. Gehör; 37, pp 181-185.

Soultanian, Nataliya (2012): Wie russische Kinder Deutsch lernen. Sprachförderung in der Familie und im Kindergarten, Tübingen: Francke.

Švedova, Natalija (2005): Russkaja grammatika. 1. Fonetika, fonologija, udarenije, intonacija, slovoobrazovanije, morfologija, Moskva: Nauka.

Thoma, Dieter/Tracy, Rosemarie (2006): Deutsch als frühe Zweitsprache: zweite Erstsprache? In: Ahrenholz, Bernd (ed.), Kinder mit Migrationshintergrund - Spracherwerb und Fördermöglichkeiten, Freiburg: Fillibach, pp 58-79.

Tracy, Rosemarie/Gawlitzek-Maiwald, I. (2000). Bilingualismus in der frühen Kindheit. In: Grimm, H. (ed.), Enzyklopädie der Psychologie, Bd.3, Sprachentwicklung, Göttingen: Hogrefe, pp 495-535.

Tracy, Rosemarie (2002): Deutsch als Erstsprache: Was wissen wir über die wichtigsten Meilensteine des Erwerbs? In: Universität Mannheim. Informationsbroschüre 1/2002 der Forschungs- und Kontaktstelle Mehrsprachigkeit, Mannheim: Universität Mannheim. 